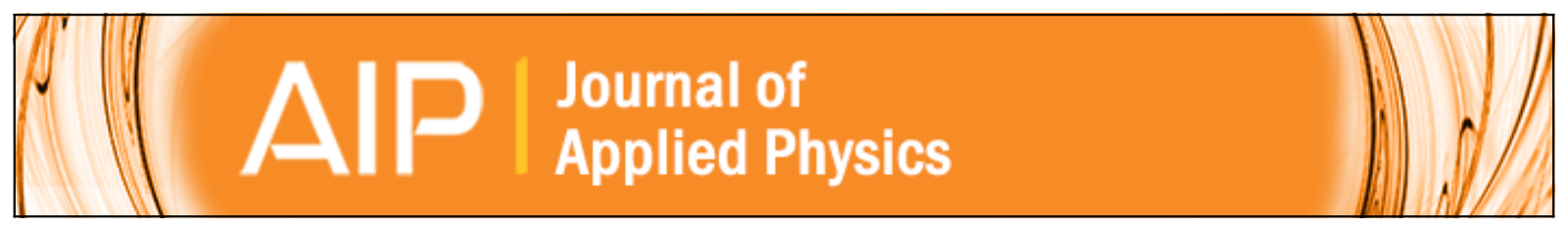

\title{
Influence of Ge addition on the magnetocaloric effect of a Co-containing Nanoperm- type alloy
}

V. Franco, J. S. Blázquez, and A. Conde

Citation: Journal of Applied Physics 103, $07 B 316$ (2008); doi: 10.1063/1.2835688

View online: http://dx.doi.org/10.1063/1.2835688

View Table of Contents: http://scitation.aip.org/content/aip/journal/jap/103/7?ver=pdfcov

Published by the AIP Publishing

\section{Articles you may be interested in}

Influence of $\mathrm{Mn}$ on the magnetocaloric effect of nanoperm-type alloys

J. Appl. Phys. 108, 073921 (2010); 10.1063/1.3489990

Influence of $\mathrm{Co}$ and $\mathrm{Ni}$ addition on the magnetocaloric effect in $\mathrm{Fe} 88-2 \times \mathrm{Co} \times \mathrm{Ni}$ x $\mathrm{Zr} 7 \mathrm{~B} 4 \mathrm{Cu} 1$ soft magnetic amorphous alloys

Appl. Phys. Lett. 96, 182506 (2010); 10.1063/1.3427439

The magnetocaloric effect in soft magnetic amorphous alloys

J. Appl. Phys. 101, 09 C503 (2007); 10.1063/1.2709409

The influence of Co addition on the magnetocaloric effect of Nanoperm-type amorphous alloys J. Appl. Phys. 100, 064307 (2006); 10.1063/1.2337871

Influence of Co addition on the magnetocaloric effect of FeCoSiAIGaPCB amorphous alloys Appl. Phys. Lett. 88, 132509 (2006); 10.1063/1.2188385

\section{SHIMADZU Powerful, Multi-functional UV-Vis-NIR and Excellence in science FTIR Spectrophotometers}

Providing the utmost in sensitivity, accuracy and resolution for applications in materials characterization and nano research

- Photovoltaics

- Polymers

- Thin films

- Paints

- Ceramics

- DNA film structures

- Coatings

- Packaging materials
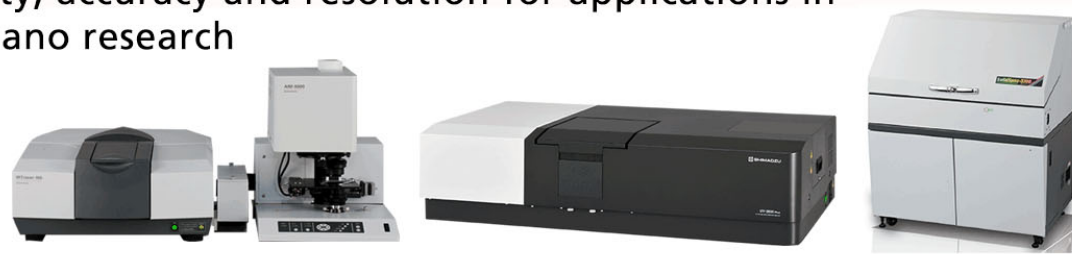


\title{
Influence of Ge addition on the magnetocaloric effect of a Co-containing Nanoperm-type alloy
}

\author{
V. Franco, ${ }^{\text {a) }}$ J. S. Blázquez, and A. Conde \\ Departamento Física de la Materia Condensada, ICMSE-CSIC, Universidad de Sevilla, P.O. Box 1065, \\ 41080 Sevilla, Spain
}

(Presented on 7 November 2007; received 18 August 2007; accepted 9 November 2007; published online 30 January 2008)

The influence of the partial substitution of $\mathrm{B}$ by $\mathrm{Ge}$ on the magnetocaloric response of $\mathrm{Fe}_{78} \mathrm{Co}_{5} \mathrm{Zr}_{6} \mathrm{~B}_{10} \mathrm{Cu}_{1}$ is studied. Ge addition produces a reduction in the temperature at which the peak entropy change takes place, as well as a slight decrease in the magnitude of the peak, $\left|\Delta S_{M}^{\mathrm{pk}}\right|$. The refrigerant capacity, $\mathrm{RC}$, and its field dependence is also analyzed: although Ge addition increases $\mathrm{RC}$ of the Co-containing alloy, the largest RC value corresponds to the Co- and $\mathrm{Ge}$-free alloy. This will be discussed on the basis of the recently proposed universal curve for the magnetic entropy change, which is also followed by the $\mathrm{FeZrBCu}(\mathrm{Co}, \mathrm{Ge})$ alloy series. (C) 2008 American Institute of Physics. [DOI: 10.1063/1.2835688]

Research on the magnetocaloric effect (MCE) is taking an increasing attention from the scientific community as its application in magnetic refrigeration connects with the growing demand of environmental friendly and energetically efficient technologies. ${ }^{1-3}$ Current magnetic refrigerator prototypes are mainly based on rare-earth based materials, ${ }^{4,5}$ most of them with a magnetocaloric response associated to a second order magnetic phase transition. With the aim of reducing materials costs, soft magnetic amorphous alloys have been recently proposed as low cost candidates for high temperature magnetic refrigeration. ${ }^{6-13}$ Albeit the maximum magnetic entropy change $\left|\Delta S_{M}^{\mathrm{pk}}\right|$ for these alloys is modest when compared to that of rare-earth-based materials, ${ }^{14,15}$ their refrigerant capacity, $\mathrm{RC}$, is in some cases comparable to that of the latter ones. ${ }^{13}$ Moreover, their reduced magnetic hysteresis (virtually negligible), higher electrical resistivity (which would decrease eddy current losses), tunable Curie temperature, and, in the case of bulk amorphous alloys, outstanding mechanical properties are beneficial characteristics for a successful application of the material.

Apart from studying the peculiarities of the different alloy series, a current line of research consists in studying the field dependence of the magnetic entropy change $\Delta S_{M}{ }^{16}$ As the performance of a magnetic refrigerator depends on the maximum applied field, the analysis of the field dependence of $\Delta S_{M}$ for different types of materials can give further clues to improve the performance of refrigerant materials for the magnetic field range employed in actual refrigerators (generally, 10-20 kOe). It has been recently shown that materials with a second order magnetic phase transition, ranging from soft magnetic amorphous alloys to lanthanides, follow a universal curve for $\Delta S_{M}$ (Refs. 12,17, and 18) when measured up to different maximum applied fields. However, although the relevant characteristic of a magnetocaloric material should be a compromise between the value of the peak entropy change and the width of the maximum (usually repre-

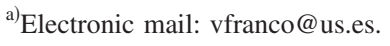

sented by the refrigerant capacity), up to now smaller attention has been paid to the field dependence of RC. The aim of this work is twofold: from the materials science point of view, the MCE characteristics of the $\mathrm{FeZrBCu}(\mathrm{Co}, \mathrm{Ge})$ alloy series will be presented. As recently shown, ${ }^{12}$ the different alloying additives which allow controlling the Curie temperature of the material can have a different influence on the magnitude of the peak entropy change. Ge is used with the aim of tuning the Curie temperature of the Co-containing Nanoperm-type alloy without decreasing too much the magnetic entropy change. From a more fundamental point of view, the field dependence of both $\Delta S_{M}$ and RC will be analyzed on the basis of the universal curve. Although a comparison between the Co-containing and Co-free alloys was previously presented, ${ }^{19}$ some of the results will also be referred in this work in order to analyze the scaling of the properties of the full series.

Amorphous ribbons $(\sim 5 \mathrm{~mm}$ wide and $20-30 \mu \mathrm{m}$ thick) of $\mathrm{Fe}_{78} \mathrm{Co}_{5} \mathrm{Zr}_{6} \mathrm{~B}_{5} \mathrm{Ge}_{5} \mathrm{Cu}_{1}, \mathrm{Fe}_{78} \mathrm{Co}_{5} \mathrm{Zr}_{6} \mathrm{~B}_{10} \mathrm{Cu}_{1}$, and $\mathrm{Fe}_{83} \mathrm{Zr}_{6} \mathrm{~B}_{10} \mathrm{Cu}_{1}$ were obtained by melt spinning. The amorphous character of the as-quenched alloys was checked by $\mathrm{x}$-ray diffraction. The field dependence of magnetization was measured in a Lakeshore 7407 vibrating sample magnetometer using a maximum applied field $H=15 \mathrm{kOe}$ with field steps of $50 \mathrm{Oe}$, for constant temperatures in the range of $300-625 \mathrm{~K}$ with increments of $10 \mathrm{~K}$. Prior to the measurements, the stress of the samples was relaxed by preannealing. Details about the devitrification process of these alloys were given elsewhere. $^{20}$

The magnetic entropy change due to the application of a magnetic field $H$ has been evaluated from the processing of the temperature and field dependent magnetization curves using a numerical approximation to the equation

$$
\Delta S_{M}=\int_{0}^{H}\left(\frac{\partial M}{\partial T}\right)_{H} d H
$$

where the partial derivative is replaced by finite differences and the integration is performed numerically. 


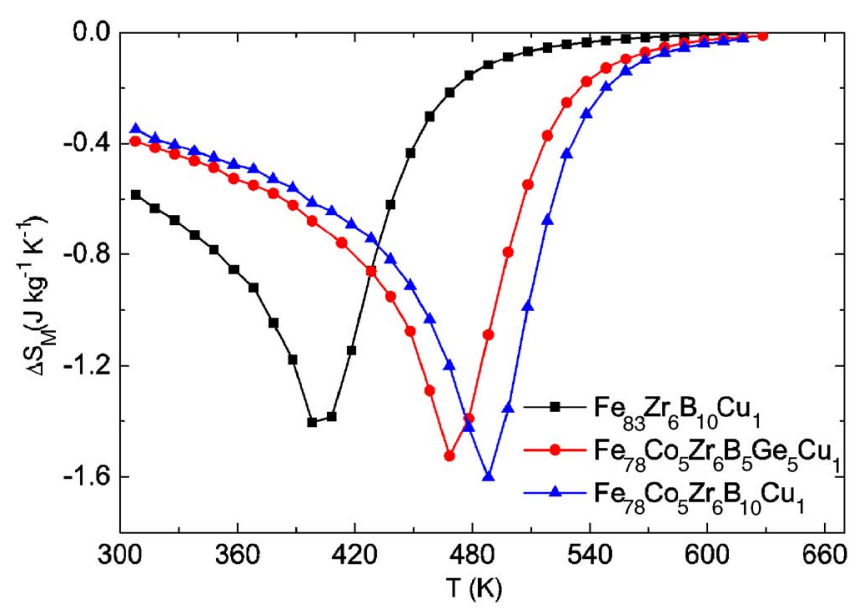

FIG. 1. (Color online) Temperature dependence of the magnetic entropy change measured for a maximum applied field of $15 \mathrm{kOe}$. Lines are a guide to the eye.

There are different definitions of RC in the literature. Among the most extended ones is that given by Wood and Potter, $^{21}$ in which the RC of a reversible refrigeration cycle operating between $T_{h}$ and $T_{c}$ (the temperatures of the hot and cold reservoirs, respectively) is defined as $\mathrm{RC}_{\mathrm{WP}}=\left|\Delta S_{M}\right|\left(T_{h}\right.$ $-T_{c}$ ), where $\left|\Delta S_{M}\right|$ is the magnetic entropy change at the hot and cold ends of the cycle and the optimal refrigeration cycle is that which maximizes this magnitude. Another common definition of $\mathrm{RC}$ is the product of the peak entropy change times the full width at half maximum of the peak, $\Delta T$, $\mathrm{RC}_{\mathrm{FWHM}}=\left|\Delta S_{M}^{\mathrm{pk}}\right| \Delta T$. The second version of this magnitude is calculated in this work.

Figure 1 shows the $\Delta S_{M}(T)$ curves for the three studied alloys for a maximum applied field of $15 \mathrm{kOe}$. Although $\mathrm{Ge}$ addition decreases the temperature at which the peak takes place, $T_{\mathrm{pk}}$, with respect to the Co-containing, Ge-free alloy, it also slightly decreases $\left|\Delta S_{M}^{\mathrm{pk}}\right|$. Recent calculations indicate that it is possible to construct a universal curve for a series of alloys with the help of a single reference temperature, ${ }^{22}$ instead of using two reference temperatures as proposed in the initial phenomenological approach. ${ }^{12,17,18}$ In fact, provided that the magnetic equation of state for the studied material is known (and that the only magnetic transition is a Curie transition), the magnetization curve for the material could be described with a single set of parameters for the whole temperature range. In the case of the Arrott-Noakes equation of state, $^{23}$

$$
H^{1 / \gamma}=a\left(T-T_{\text {Curie }}\right) M^{1 / \gamma}+b M^{1 / \beta+1 / \gamma},
$$

these parameters are the critical exponents, the Curie temperature, $a$, and $b$. As the critical exponents of an alloy are given by its universality class, three additional free parameters, characteristic of the alloy, remain: $a, b$, and the Curie temperature. Therefore, the universal curve for a series of alloys of the same universality class should be constructed with such a number of variables: $T_{\mathrm{pk}},\left|\Delta S_{M}^{\mathrm{pk}}\right|$ and one single reference temperature, $T_{r}$. This prediction is confirmed for experimental data in Fig. 2, where the universal curve is constructed by normalizing the $\Delta S_{M}(T)$ curves with respect to their peak and by scaling the temperature axis with $\theta$

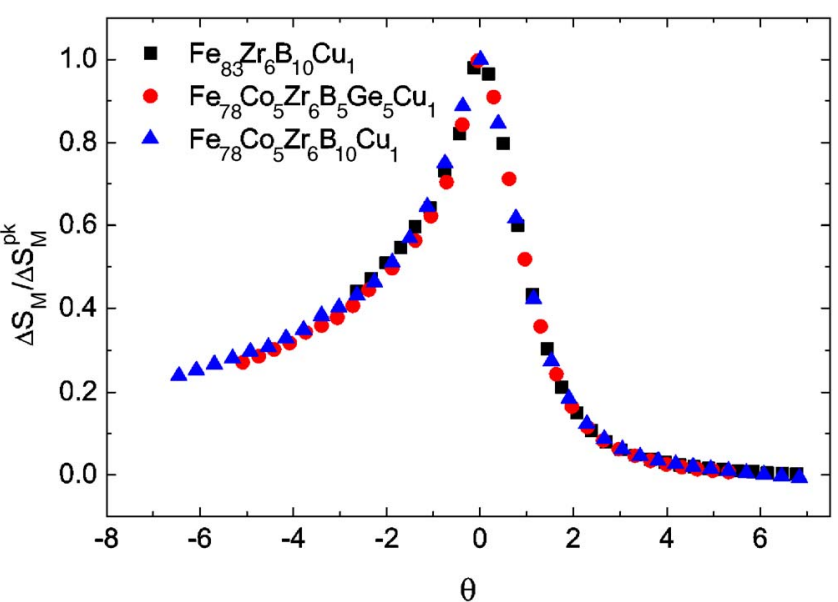

FIG. 2. (Color online) Universal curve constructed with a single reference temperature for the three studied compositions.

$=\left(T-T_{\mathrm{pk}}\right) /\left(T_{r}-T_{\mathrm{pk}}\right)$, where $T_{r}$ has been selected as the temperature corresponding to $\Delta S\left(T_{r}\right)=0.5 \Delta S_{M}^{\mathrm{pk}}$ above the peak temperature.

The field dependence of $\mathrm{RC}_{\mathrm{FWHM}}$ for the Ge containing alloy is presented in Fig. 3. The curves for the other two compositions are similar. A power law has been fitted to the experimental data, indicating that a single field exponent can be found. Taking into account that the peak entropy change scales with field as ${ }^{17}$

$$
\Delta S_{M} \propto H^{1+(1 / \delta)(1-1 / \beta)},
$$

and that the reference temperature scales with field as ${ }^{22}$

$$
T_{r} \propto H^{1 / \beta \delta},
$$

the product of the peak entropy change times the full width at half maximum, i.e., $\mathrm{RC}_{\mathrm{FWHM}}$, should scale with field as a power law with an exponent $1+1 / \delta$, with $\delta$ being the critical exponent of the magnetic transition. The exponent obtained from the fit of $\mathrm{RC}_{\mathrm{FWHM}}$ of all the three studied compositions, $1.20 \pm 0.03$, is compatible with the previously reported value $^{17}$ of the critical exponent for this alloy series of $\delta$ $=4.329 \pm 0.002$. It is worth mentioning that the field dependence of $\mathrm{RC}_{\mathrm{WP}}$, although following a potential law, has a

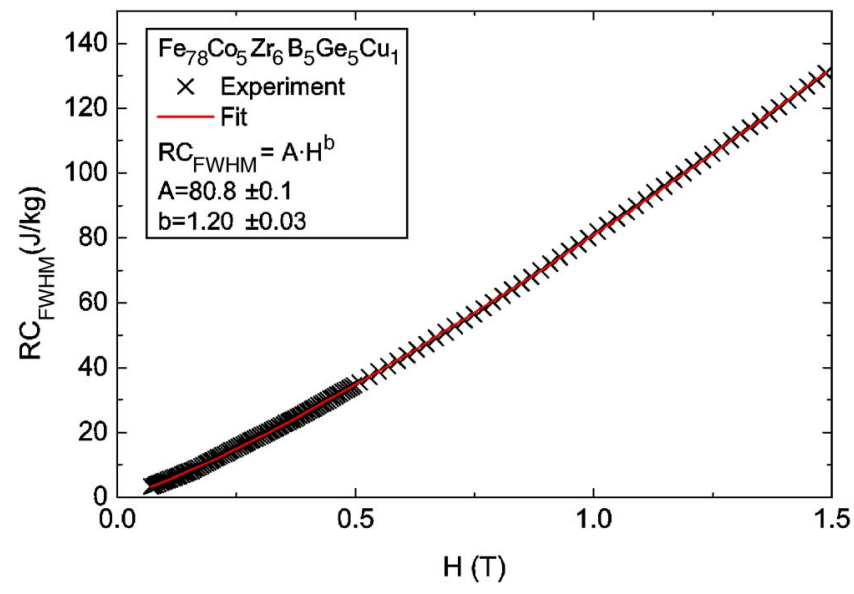

FIG. 3. (Color online) Field dependence of the refrigerant capacity of the Ge-containing alloy. Line corresponds to the power law fitting of the data. 


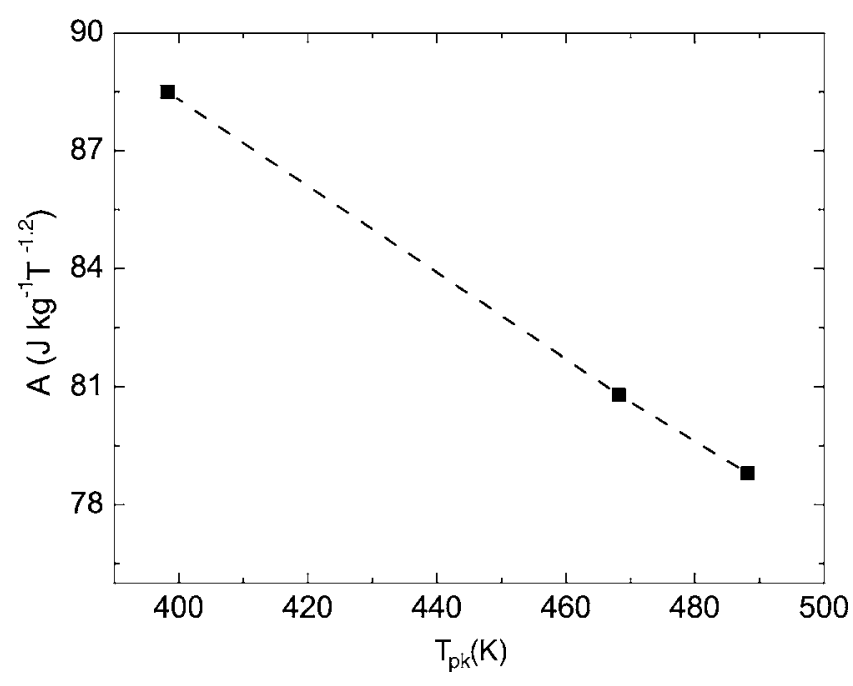

FIG. 4. Relationship between the peak temperature and the prefactor of the power law fit of $\mathrm{RC}_{\mathrm{FWHM}}$. Lines are a guide to the eye.

different field exponent. This difference can be justified by the nonlinear shape of the curves below and above the peak, and further studies are being undertaken to correlate the experimental values with theoretical predictions.

Figure 4 corresponds to the prefactor of the previously mentioned fit of $\mathrm{RC}_{\mathrm{FWHM}}$. Although $\mathrm{Ge}$ addition increases $\mathrm{RC}_{\mathrm{FWHM}}$ with respect to the Co-containing Ge-free alloy, the highest refrigerant capacity is obtained for the $\mathrm{Ge}$ - and $\mathrm{Co}-$ free alloy.

In conclusion, the magnetocaloric response of amorphous ribbons of the $\mathrm{FeZrBCu}(\mathrm{Co}, \mathrm{Ge})$ alloy series has been presented. Partial substitution of $\mathrm{B}$ by $\mathrm{Ge}$ in the Cocontaining alloy can be used to tune the Curie temperature of the Co-containing alloy and to increase its refrigerant capacity, at the expense of a small reduction of the peak entropy change. This alloy series follows the recently proposed universal curve for $\Delta S_{M}$, which can be calculated with the help of a single reference temperature. The field dependence of $\mathrm{RC}_{\mathrm{FWHM}}$ has been also studied, following a power law with an exponent value compatible with previous theoretical predictions.
This work was supported by the Spanish Government and EU-FEDER (Project Nos. MAT 2004-04618 and MAT 2007-65227) and the PAI of Junta de Andalucía (Project No. P06-FQM-01823). J.S.B. is grateful to Junta de Andalucía for a research contract.

${ }^{1}$ K. A. Gschneidner, Jr. and V. K. Pecharsky, Annu. Rev. Mater. Sci. 30, 387 (2000)

${ }^{2}$ A. M. Tishin and Y. I. Spichkin, The Magnetocaloric Effect and Its Applications (Institute of Physics, Bristol, 2003).

${ }^{3}$ E. Brück, J. Phys. D 38, R381 (2005).

${ }^{4}$ C. A. Zimm, A. Jastrab, A. Sternberg, V. K. Pecharsky, K. A. Gschneidner, Jr., M. G. Osborne, and I. E. Anderson, Adv. Cryog. Eng. 43, 1759 (1998).

${ }^{5}$ K. A. Gschneidner, Jr. and V. K. Pecharsky, Proceedings of the Second International Conference on Magnetic Refrigeration at Room Temperature, IIF-IIR, Paris, 2007 (unpublished), pp. 9-21.

${ }^{6}$ D. Wang, K. Peng, B. Gu, Z. Han, S. Tang, W. Qin, and Y. Du, J. Alloys Compd. 358, 312 (2003).

${ }^{7}$ S. Atalay, H. Gencer, and V. S. Kolat, J. Non-Cryst. Solids 351, 2373 (2005).

${ }^{8}$ S. G. Min, K. S. Kim, S. C. Yu, H. S. Suh, and S. W. Lee, J. Appl. Phys. 97, 10M310 (2005).

${ }^{9}$ V. Franco, J. S. Blázquez, C. F. Conde, and A. Conde, Appl. Phys. Lett. 88, 042505 (2006)

${ }^{10}$ F. Johnson and R. D. Shull, J. Appl. Phys. 99, 08 K909 (2006).

${ }^{11}$ Q. Luo, D. Q. Zhao, M. X. Pan, and W. H. Wang, Appl. Phys. Lett. 89, 081914 (2006).

${ }^{12}$ V. Franco, J. S. Blázquez, M. Millán, J. M. Borrego, C. F. Conde, and A. Conde, J. Appl. Phys. 101, 09C503 (2007).

${ }^{13}$ V. Franco, C. F. Conde, A. Conde, and L. F. Kiss, Appl. Phys. Lett. 90, 052509 (2007).

${ }^{14}$ V. K. Pecharsky and K. A. Gschneidner, Jr., Phys. Rev. Lett. 78, 4494 (1997).

${ }^{15}$ V. Provenzano, A. J. Shapiro, and R. D. Shull, Nature (London) 429, 853 (2004).

${ }^{16}$ A. M. Tishin, A. V. Derkach, Y. I. Spichkin, M. D. Kuz'min, A. S. Chernyshov, K. A. Gschneidner, Jr., and V. K. Pecharsky, J. Magn. Magn. Mater. 310, 2800 (2007).

${ }^{17}$ V. Franco, J. S. Blázquez, and A. Conde, Appl. Phys. Lett. 89, 222512 (2006).

${ }^{18}$ V. Franco, A. Conde, V. K. Pecharsky, and K. A. Gschneidner, Jr., Europhys. Lett. 79, 47009 (2007).

${ }^{19}$ V. Franco, J. S. Blázquez, and A. Conde, J. Appl. Phys. 100, 064307 (2006).

${ }^{20}$ J. S. Blázquez, S. Roth, C. Mickel, and A. Conde, Acta Mater. 53, 1241 (2005).

${ }^{21}$ M. E. Wood and W. H. Potter, Cryogenics 25, 667 (1985).

${ }^{22}$ V. Franco, A. Conde, J. M. Romero-Enrique, and J. S. Blázquez (unpublished).

${ }^{23}$ A. Arrott and J. E. Noakes, Phys. Rev. Lett. 19, 786 (1967). 